\title{
QUALIDADE DE VIDA DOS PAIS, DOADORES OU NÃO DE RIM, E ASPECTOS BIOPSICOSSOCIAIS DO TRANSPLANTE PEDIÁTRICO
}

\author{
Quality of life of parents, kidney donors or not, and biopsychosocial aspects of pediatric transplant
}

\author{
Cláudia Hilgert', Gibsi Rocha', Ana Maria Verçoza², Norma Escosteguy', Márcia Coutinho', Evelyn Vigueras³, \\ Larissa Ferreira Rios', Lauro Toffolo', Martina Cezar Kopittke', Mayara Mayer e Vinicius Correa da Luz'
}

\begin{abstract}
RESUMO
Objetivo: Avaliar a qualidade de vida de genitores doadores e não-doadores de rim e os aspectos biopsicossociais envolvidos no transplante renal de pacientes pediátricos. Métodos: Estudo seccional-transversal, a amostra foi composta por 61 genitores de pacientes transplantados que fazem acompanhamento regular no ambulatório de nefrologia pediátrica do HSL/PUC-RS, sendo 15 doadores e 46 não-doadores, acompanhados por seis meses. Os entrevistados responderam a dois questionários elaborados pelos autores e ao questionário SF-36. A análise estatística foi realizada através das tabelas de contingências, teste qui-quadrado, Mann-Whitney e Monte Carlo para obter a significância de 5\% do qui-quadrado. Resultados: A qualidade de vida de doadores e não-doadores apresentaram as seguintes medianas nos oito domínios do SF 36: Capacidade Funcional 92/90; Aspecto Físico 100/100; Dor 72/72; Estado Geral de Saúde 89/82; Vitalidade 60/65; Aspecto Social 100/87; Aspecto Emocional 100/100 e Saúde Mental 68/68. Não houve diferença significativa entre a qualidade de vida de doadores e não-doadores. Conclusão: Não houve diferença entre a qualidade de vida de doadores de rim e não-doadores, o que não exclui a necessidade de se estabelecer programas que estimulem a doação intervivos.
\end{abstract}

Descritores: Qualidade de Vida, Transplante Renal, Doadores Vivos, Relações Pais-Filho.

\footnotetext{
Instituições:

1 Departamento de Psiquiatria do Hospital São Lucas da Pontifícia Universidade Católica do Rio Grande do Sul - Porto Alegre / RS - Brasil

2 Departamento de Nefrologia Pediátrica do Hospital São Lucas da Pontifícia Universidade Católica do Rio Grande do Sul - Porto Alegre / RS - Brasil

${ }^{3}$ Departamento de Psicologia do Hospital São Lucas da Pontifícia Universidade Católica do Rio Grande do Sul - Porto Alegre / RS - Brasil
}

Correspondência:

Cláudia Hilgert

Rua Dário Pederneiras, 16 / 701 - Porto Alegre / RS - CEP: 90630-090 - Brasil

Tel.: (51) 99033162

E-mail: clauhilg@terra.com.br

Recebido em: 18.03 .2009

Aceito em: 06.04.2009

\section{INTRODUÇÃO}

Crianças e adolescentes com doença renal apresentam atualmente opções terapêuticas mais eficazes do que no passado. Entre as opções existentes estão a diálise (hemodiálise, diálise peritoneal) e o transplante renal, que ainda parece ser o tratamento de escolha para esses pacientes, em função de propiciar o crescimento, desenvolvimento e reabilitação mais efetivos. ${ }^{1}$ Entretanto, o processo de transplante de órgãos, seja de origem cadavérica ou intervivos, causa grande impacto na família inteira. ${ }^{2,3}$

Algumas vantagens do transplante renal incluem término dos problemas de acesso vascular, melhora nutricional, liberalização da restrição de fluídos, diminuição de doenças ósseas e melhor ajuste psicossocial. ${ }^{4} \mathrm{O}$ prognóstico do rim transplantado varia de acordo com o tipo de doador. Se o transplante for feito de doador vivo, a sobrevida do enxerto após a cirurgia é significativamente melhor do que de doador falecido. Essa maior sobrevida do rim enxertado é de grande importância para a criança que tem longa expectativa de vida pela frente. Quando o rim é de doador vivo, existe também outra vantagem adicional, como a possibilidade de planejar e até antecipar o transplante, realizado, assim, de uma forma eletiva. ${ }^{5}$

Estudos demonstram que os pais enfrentam um estresse psicossocial acentuado ao se adaptarem a uma vida com uma criança cronicamente doente. ${ }^{6,7}$ Após o diagnóstico de uma doença progressiva e possivelmente fatal como a insuficiência renal, levanta-se a questão sobre o transplante do órgão. Então, os pais precisam enfrentar o dilema de doar ou não um de seus próprios rins. ${ }^{2}$ 
Seria ético questionar os pais sobre fazer ou não a doação em tal situação? Mas, por outro lado, seria justificável não lhes colocar a questão e assim privá-los da chance de ajudar o filho doente? Essas são perguntas que vêm recebendo atenção por parte de pesquisadores interessados na ética envolvida com a doação de órgãos intervivos..$^{2-9}$

O número de transplantes renais tem aumentado consideravelmente nos últimos anos em todo o mundo e esse aumento é observado predominantemente no transplante com órgão de doador falecido. Esse evento é particularmente evidenciado nos EUA, em que o número dos transplantes intervivos familiares tem se mantido o mesmo ao longo dos anos, enquanto o dos transplantes de doador falecido dobrou. O percentual de transplantes vivos familiares é em torno de $20 \%$ neste país. Na Europa, especialmente por característica da legislação dos países, a ênfase é para o transplante de doador falecido $(85 \%){ }^{1}$

Atualmente, existe no Brasil legislação específica para regulamentação da disponibilização de órgãos para transplante, qualquer que seja o doador. Os transplantes com doadores vivos podem ser feitos com indivíduos consangüíneos, familiares até o $4^{\circ}$ grau, embora mais comumente os doadores sejam parentes próximos, como pais e irmãos. Os transplantes com doadores vivos são realizados em caráter eletivo com doador disponível ou preferencialmente com o de melhor compatibilidade em relação aos antígenos do complexo HLA. ${ }^{10}$

Tendo em vista esses fatos, surgiu o interesse dos pesquisadores do presente estudo em avaliar as consequências físicas, psicológicas, sociais e o impacto na qualidade de vida que a doação e o transplante exercem nos pais envolvidos nessa questão.

\section{MÉTODOS}

Este é um estudo seccional-transversal. A pesquisa foi realizada com genitores de pacientes transplantados que fazem acompanhamento regular no Serviço de Nefrologia Pediátrica do Hospital São Lucas da PUC-RS. Esse serviço atende pelo SUS atualmente 38 pacientes transplantados, sendo que apenas 61 genitores (15 doadores e 46 não-doadores) de 31 desses pacientes aceitaram participar dessa pesquisa. Os transplantes ocorreram entre 1980 e 2007 e a idade dos pacientes na época do primeiro transplante variou de 01 a 19 anos. Esses pacientes fazem revisões ambulatoriais com a equipe de nefrologia pediátrica que podem ser desde semanais até trimestrais.

Questionários individuais foram entregues aos genitores, doadores e não-doadores, quando esses acompanhavam seus filhos nas consultas ambulatoriais durante os meses de setembro de 2007 a março de 2008 .

Os genitores que decidiram participar da pesquisa foram informados primeiramente sobre os objetivos do estudo e a confidencialidade dos dados, assinando então o termo de consentimento livre e esclarecido, que abordou todas as questões sobre o projeto. No termo de consentimento constaram: objetivos, justificativa, procedimentos, garantias individuais, contribuições da pesquisa e telefone do pesquisador responsável. O mesmo foi assinado pelo participante, que recebeu uma cópia. Não foram incluídos no estudo indivíduos com distúrbios mentais ou de consciência, incapazes de responder aos questionamentos ou que não concordaram com os termos do estudo.
Um dos questionários entregue foi elaborado pelos autores e continha questões baseadas no inquérito feito por Kärrfelt et al. (1998) em seu trabalho "To be or not to be a living donor", já que inexistem questionários específicos validados no Brasil sobre qualidade de vida e aspectos biopsicossociais de doadores de rim. As questões referiamse a dados pessoais, demográficos e clínicos dos entrevistados e também a questões sobre a decisão quanto à doação renal, a cirurgia, aspectos perioperatórios na visão dos pais, relação dos pais entre si, relação entre os pais e a criança transplantada, cuidados psicossociais, conseqüências sociais do transplante e sintomas psiquiátricos. Esse inquérito foi feito para ambos os grupos de genitores: doadores e não-doadores. Em outro questionário, as perguntas foram mais específicas para cada grupo.

A maioria das questões fornecia três alternativas como respostas: "muito", "mais ou menos" ou "nem um pouco". Algumas também forneciam cinco alternativas para resposta: melhorou muito, pouco, não mudou, piorou um pouco ou muito.

Para avaliar a qualidade de vida utilizou-se o questionário SF-36 (Medical Outcomes Study 36 - Item Short-Form Health Survey). Esse é um instrumento genérico de avaliação de qualidade de vida de fácil administração e compreensão e de rápida aplicação. É um questionário multidimensional formado por 36 itens, englobados em oito escalas ou componentes: capacidade funcional, aspectos físicos, dor, estado geral da saúde, vitalidade, aspectos sociais, emocionais e saúde mental. Apresenta um escore final de 0 a 100, onde zero corresponde ao pior estado geral de saúde e 100 ao melhor estado de saúde. Foi validado para uso no Brasil por Ciconelli et al. em 1999. ${ }^{11}$

Os questionários foram lidos pelos pesquisadores para que não houvesse distinção entre pacientes analfabetos e alfabetizados. A leitura foi feita de forma pausada, para que os pacientes pudessem escolher a melhor alternativa para cada questão. Quando o entrevistado não entendia o significado de alguma pergunta, o entrevistador lia a pergunta novamente de forma mais lenta, sem utilizar outras palavras, evitando assim a modificação do sentido original. A aplicação dos questionários e a explicação sobre os objetivos do trabalho tiveram uma duração média de 35 minutos.

O banco de dados foi construído em Excel e a análise estatística realizada no programa SPSS, versão 11.5. As técnicas estatísticas consistiram de tabelas de contingências, testes qui-quadrado e Mann-Whitney. Devido ao pequeno tamanho amostral de doadores, foi utilizado o método de Monte Carlo para obtenção de significância do teste qui-quadrado de $5 \%$.

Os dados obtidos dos entrevistados foram mantidos em banco de dados sigiloso e os resultados tornados públicos, independente de terem sido favoráveis ou não.

Este projeto de pesquisa foi protocolado pelo Comitê de Ética em Pesquisa da Pontifícia Universidade Católica-RS (PUCRS). O estudo não ofereceu riscos, e seguiu as diretrizes e normas regulamentadoras de pesquisas em seres humanos, conforme a resolução 196/96 do Conselho Nacional de Saúde. Aqueles indivíduos que não aceitaram ou por algum motivo não puderam participar do estudo continuaram sendo atendidos normalmente nos ambulatórios, sem qualquer prejuízo.

\section{RESULTADOS}

Quanto às principais características da amostra total (doadores e não-doadores): $62 \%$ dos genitores eram do sexo feminino; $82 \%$ da 
raça branca; $65 \%$ casados; $28 \%$ eram do lar, $28 \%$ profissionais liberais e $26 \%$ possuíam outro tipo de vínculo empregatício (Gráfico 1). $\mathrm{Na}$ época que responderam os questionários, a média de idade dos genitores doadores era de 36,6 anos e de não-doadores de 44,8. A escolaridade dos doadores foi de 9,4 anos de estudo versus 7,9 anos do grupo de não-doadores.

A respeito da doação ou não do órgão, 93\% da amostra total de genitores sentiram-se livres para decidir e $87 \%$ acharam que tiveram tempo suficiente para tomar essa decisão. As informações médicas foram satisfatórias para $88 \%$ da amostra geral e $94 \%$ não se sentiram pressionados durante a tomada da decisão. Sintomas como tristeza e ansiedade antes do transplante ocorreram em $66 \%$ da amostra total, sendo que destes, $73 \%$ eram do grupo de pais não-doadores $(\mathrm{P}=0,084)$. Alguns dias após o transplante, esses sintomas continuaram para $42 \%$ da amostra total e entre o grupo de nãodoadores foi de $48 \%(\mathrm{P}=0,059)$. Na amostra geral, $81 \%$ dos genitores sentiram-se mais satisfeitos consigo mesmos após o transplante e $45 \%$ notaram melhora na relação com o filho transplantado, relatando em $33 \%$ dos casos que se sentiram mais unidos.

No grupo de doadores, $66 \%$ eram do sexo feminino. 53\% disseram ter tomado essa atitude por causa da urgência da situação e $26 \%$ por causa do sofrimento que o filho estava passando. Para $40 \%$ do total

Gráfico 1. Principais características da amostra total (doadores e não-doadores)
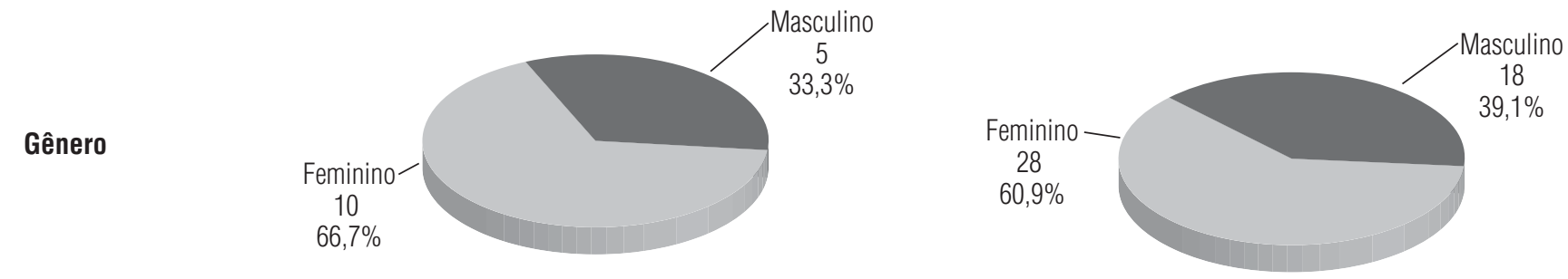

Estado Civil
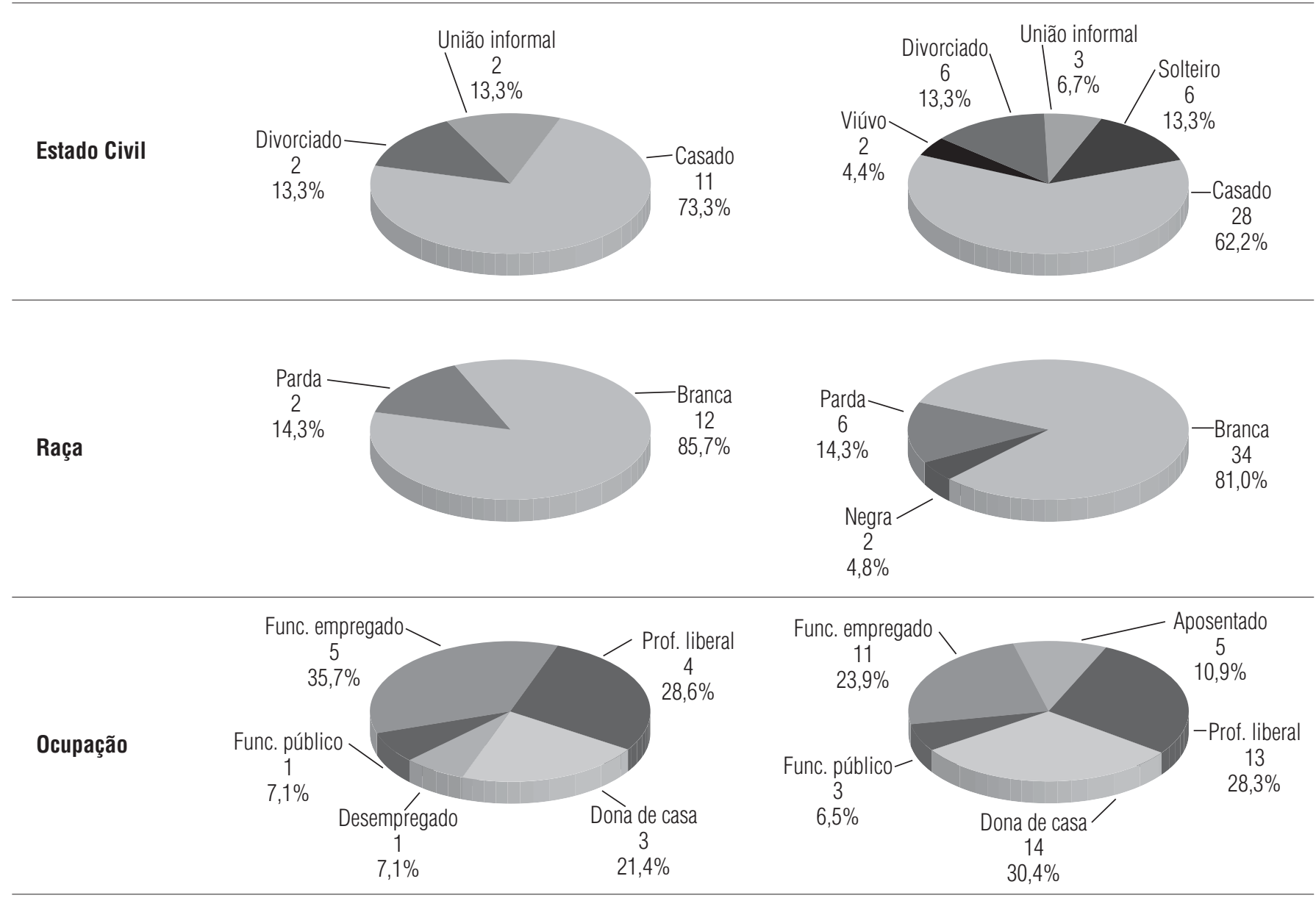
de doadores, a dor no pós-operatório não foi maior que o esperado, mas para 33\% foi muito maior. O cuidado médico recebido após o transplante foi muito maior para $46 \%$ dos doadores, para $20 \%$ foi um pouco maior e em $26 \%$ não houve mudança. Para $46 \%$ dos doadores houve grande aumento na satisfação própria após o transplante e 93\% relataram que fariam tudo de novo se fosse necessário.

No grupo de não-doadores $30 \%$ tinham condições médicas para doar, porém, o que os motivou a não doar o órgão não foi informado em 56\% dos casos. Para 17\% deles os exames solicitados pela equipe para descobrir a compatibilidade não foram feitos e outros $17 \%$ foram aconselhados pelos médicos que acompanhavam o caso a não se submeterem à doação, já que eram portadores de problemas de saúde que contra-indicavam a cirurgia. Apenas 8,7\% dos nãodoadores não eram compatíveis e, portanto, não poderiam doar seu rim. Nesse grupo, 69\% dos transplantes realizados foram de origem cadavérica, $13 \%$ o pai foi o doador, $13 \%$ a mãe e $4,3 \%$ não informaram quem foi o doador. Nesse mesmo grupo, $73 \%$ prefeririam ter sido os doadores, porém, $76 \%$ ficaram satisfeitos com a decisão tomada e $71 \%$ afirmaram que fariam tudo novamente, se fosse preciso. Desses doadores, $43 \%$ perceberam ter recebido muito mais atenção da equipe médica após o transplante.

Quanto à qualidade de vida (Tabela 1), os doadores e não-doadores apresentaram as seguintes médias nos oito domínios do SF-36: Capacidade Funcional 92,5/90 ( $\mathrm{p}=0,617)$; Aspecto Físico 100/100 $(\mathrm{p}=0,891)$; Dor 72/72 ( $\mathrm{p}=0,989)$; Estado Geral de Saúde 89,5/82 $(p=0,255)$; Vitalidade $60 / 65(p=0,270)$; Aspecto Social 100/87,5 $(\mathrm{p}=0,390)$; Aspecto Emocional 100/100 $(\mathrm{p}=0,276)$ e Saúde Mental $68 / 68(\mathrm{p}=0,536)$.

\section{DISCUSSÃO}

O processo de transplante de órgãos em crianças tem grande impacto na família inteira. Alguns estudos têm demonstrado que os pais sofrem grande estresse psicossocial, já que eles têm que se adaptar à vida com uma criança cronicamente doente. , $^{2,6,7}$

Levando-se em conta as considerações éticas envolvidas nessa situação, seria esperado que alguns pais sofressem pressão externa para serem doadores, como foi demonstrado no estudo conduzido por Kärrfelt et al. (1998). Porém, na amostra que foi estudada pela presente pesquisa, a maioria dos pais relatou sentir-se livre e com tempo suficiente para decidir sobre o transplante de seus filhos. Esses pais também consideraram que a equipe médica teve papel importante no esclarecimento das condições clínicas de seus filhos e que conseguiu dar tempo suficiente para que pudessem decidir o que seria melhor, sem pressioná-los.

Uma observação importante foi que muitos doadores sentiram-se altamente satisfeitos consigo mesmos após o transplante e que a maioria faria tudo novamente, se fosse necessário. Estudos similares também referiram esses mesmos achados, além de evidências sobre a boa qualidade de vida em curto e longo prazo. ${ }^{2,6,12,13}$

Outro dado que chamou a atenção dos pesquisadores foi que a grande maioria dos doadores era do sexo feminino, o que contraria os achados de Kärrfelt et al. (1998). Acreditamos que isso possa ter ocorrido em virtude da maioria dos questionários ter sido respondida pelas mães (62\%). Contudo, outro estudo tentou explicar porque existe maior número de doadores do sexo feminino; porém, os pesquisadores não conseguiram evidenciar essa diferença baseados apenas em perfis psicológicos e consideraram futuros estudos para que esse fato seja elucidado. ${ }^{14}$

Em geral, os pais apresentavam sintomas como tristeza e ansiedade antes e alguns dias após o transplante, o que também já foi evidenciado em outros estudos. ${ }^{2,15,16}$ Esses sintomas ocorreram mais no grupo de pais não-doadores e duraram mais tempo após o transplante. Os doadores referiram que o cuidado médico recebido após o transplante foi muito maior do que antes e notaram também aumento em sua auto-estima, além de provavelmente a família ter encorajado e admirado sua atitude. ${ }^{2,6}$ Isso também pode ter contribuído para que eles tolerassem melhor a dor após o transplante, como foi constatado.

Muitos que não doaram gostariam de ter sido doadores. O cuidado recebido da equipe médica nesse grupo foi notado como maior após o filho ter sido transplantado, mesmo que esses genitores não tivessem doado o rim. Em famílias onde o cônjuge foi doador, essa percepção de maior cuidado da equipe médica pode ter sido essencial para ajudá-los a lidar com dois doentes em casa.

Ao contrário de outros estudos, ${ }^{2,6}$ foi evidenciada melhora no relacionamento principalmente entre não-doadores e pacientes transplantados, do que com pais doadores, que não notaram diferença no relacionamento após o transplante. Isso demonstra

Tabela 1. Médias nos oito domínios do Medical Study 36 - Item Short-Form Health Survey (SF-36)

\begin{tabular}{|c|c|c|c|c|c|c|c|}
\hline \multirow[b]{3}{*}{ SF36 } & \multicolumn{6}{|c|}{ Grupo } & \multirow{3}{*}{$\frac{\text { TMW }^{*}}{\text { Valor de } p}$} \\
\hline & \multicolumn{3}{|c|}{ Doadores } & \multicolumn{3}{|c|}{ Não-doadores } & \\
\hline & P25 & Mediana & P75 & P25 & Mediana & P75 & \\
\hline Capacidade Funcional & 73,8 & 92,5 & 100 & 75 & 90,0 & 100,0 & 0,617 \\
\hline Aspecto Físico & 75,0 & 100,0 & 100,0 & 100,0 & 100,0 & 100,0 & 0,891 \\
\hline Dor & 51,0 & 72,0 & 100,0 & 51,0 & 72,0 & 84,0 & 0,989 \\
\hline Estado Geral de Saúde & 59,5 & 89,5 & 95,5 & 62,0 & 82,0 & 91,5 & 0,255 \\
\hline Vitalidade & 43,8 & 60,0 & 75,0 & 55,0 & 65,0 & 75,0 & 0,270 \\
\hline Aspecto Social & 59,4 & 100,0 & 100,0 & 62,5 & 87,5 & 100,0 & 0,390 \\
\hline Aspecto Emocional & 100,0 & 100,0 & 100,0 & 66,7 & 100,0 & 100,0 & 0,276 \\
\hline Saúde Mental & 46,0 & 68,0 & 80,0 & 56,0 & 68,0 & 80,0 & 0,536 \\
\hline
\end{tabular}

${ }^{*}$ Teste de Mann-Whitney 
que o cuidador pode receber efeitos positivos durante o processo do transplante.

Esse estudo analisou os pais e mostrou que os aspectos éticos e psicológicos da doação renal entre familiares não é um empecilho ao transplante. Assim como no trabalho de Padrão et al. (2009), também não encontramos diferença significativa entre a qualidade de vida de pais doadores e não-doadores de rim. Acreditamos que essa evidência reforça a necessidade de se estabelecer programas que estimulem a doação intervivos.

Acreditamos que os não-doadores constituam o grupo de risco psicológico com necessidades especiais de suporte nessa fase, evidenciando a necessidade de haver um suporte psicossocial mais estreito por parte da equipe de atendimento nos programas de transplante em todas etapas que contemplem tal procedimento, visando à compreensão mais abrangente dos aspectos biopsicossociais do receptor e do doador. Sendo assim, a experiência de pacientes transplantados, pais doadores e não-doadores poderia ajudar a desmistificar o procedimento intervivos e tornar esse processo mais aceitável do ponto de vista ético.

\section{CONCLUSÃO}

Não houve diferença entre a qualidade de vida de doadores de rim e não-doadores, o que não exclui a necessidade de se estabelecer programas que estimulem a doação intervivos.

\section{ABSTRACT}

Purpose: To assess the quality of life of parents kidney donors or not, and biopsychosocial aspects involved in pediatric kidney transplantation. Methods: This is a cross-sectional study. The research was conducted with 31 parents of transplanted patients regularly monitored in a pediatric nephrology outpatient clinic. It was conducted individual interviews through questionnaires answered by donors and non-donor parents. The sample was composed by 61 parents, 15 donors and 46 non-donors, evaluated for 6 months. The subjects answered two questionnaires developed by the authors and the SF-36. Statistical analysis was performed on SPSS program. Statistical techniques consisted of contingency charts, chi-square test, Mann-Whitney test and Monte Carlo to attain the 5\% significance chi-square. Results: The quality of life for donors and non donors showed the following averages in the 8 domains of the SF 36: physical function, 92/90; physical-role, 100/100, body pain, 72/72; general health, 89/82; vitality, 60/65; social function, 100/87; emotional-role, 100/100, and mental health, 68/68. There was no significant difference in the comparison between quality of life for donor and non-donor parents. Conclusion: There was no difference between quality of life of kidney donors and non donors, which does not exclude the need to set programs encouraging the living donation.

Keywords: Quality of Life, Kidney Transplantation, Living Donors, Parent-Child Relations.

\section{REFERÊNCIAS}

1. Grim PC. Pediatric renal transplantation. In: Barness LA. Advances in pediatrics. Los Angeles: Mosby-Year Book; 1992. v. 39. p. 441-93.

2. Kärrfelt HM, Berg UB, Lindblad FI, Tydén G E. To be or not to be a living donor: questionnaire to parents of children who have undergone renal transplantation. Transplantation. 1998;65(7):915-8.

3. Coutinho MA. Psychosocial Implications of individuals undergoing renal transplantation during childhood and adolescence [dissertation]. Porto Alegre: Pontifícia Universidade Católica do Rio Grande do Sul; 2003.

4. Morris PJ. Kidney transplantation: principles and practice. 4th ed. Philadelphia: WB Saunders; 1994

5. Terasaki PI, Cecka JM, Gjertson DW, Takemoto S, Cho Y W, Yuge J. Risk rate and long-term kidney transplant survival. Clinical transplants. 1996;():443-58.

6. Kamstra-Hennen L, Beebe J, Stumm S, Simmons RG. Ethical evaluation of related donations: the donor after 5 years. Transplant Proc. 1981;13:60.

7. Gouge F, Moore J Jr, Bremmer BA, McCauly CR, Johnson JP. The quality of life of donors, potential donors and recipients of living-related donor renal transplantation. Transplant Proc. 1990;22:2409.

8. Morel P, Aflmond PS. Long-term quality of life after kidney transplantation in childhood. Transplantation. 1991;52:47.
9. Shaben TR. Psychosocial issues in kidney-transplanted children and adolescents: literature review. ANNA J. 1993;20(6):663-8.

10. Manfro RC, Carvalhal GF. Symposium on transplants. Amrigs Ver. 2003;47(1):14-19.

11. Ciconelli RM, Ferraz MB, Santos W, Meinão I, Quaresma MR. Translation into Portuguese and validation of the generic questionnaire for assessing quality of life SF-36 (Brazil SF-36). J Bras Psiquiatr. 1999;39:143-150.

12. Jordan J, Sann U, Janton A, Gossmann J, Kramer W, Kachel HG, Wilhelm, Scheuermann E. Living kidney donors' long-term psychological status and health behavior after nephrectomy - a retrospective study. J Nephrol. 2004;17(5):728-35.

13. Sparta $P$, Thiel G. How living related kidney donors think about their organ donation 1 to 21 years later. Kidney Int. 1993;()44:262.

14. Achille M, Soos J, Fortin MC, Paquet M, Hébert MJ. Differences in psychosocial profiles between men and women living kidney donors. Clin Transplant. 2007;21(3):314-20.

15. Raimbault G. Psychological aspects of chronic renal failure and hemodialysis. Nephron. 1973;()11:252.

16. Brownbridge G, Fielding DM. Psychosocial adjustment to end-stage renal failure: comparing hemodialysis and transplantation. Pediatr Nephrol. 1991;5:612.

17. Padrão MB, Sens YA. Quality of life of living kidney donors in Brazil: an evaluation by the short form-36 and the WHOQOL-bref questionnaires. Clin Transplant. 2009;23(5):621. 